\title{
BMJ Open Refractive outcomes after intravitreal injection of antivascular endothelial growth factor versus laser photocoagulation for retinopathy of prematurity: a meta-analysis
}

Qihang Kong, ${ }^{1}$ Wai-kit Ming, ${ }^{2,3}$ Xue-Song Mi (D) ${ }^{1,4}$

To cite: Kong Q, Ming W, Mi X-S. Refractive outcomes after intravitreal injection of antivascular endothelial growth factor versus laser photocoagulation for retinopathy of prematurity: a meta-analysis. BMJ Open 2021;11:e042384. doi:10.1136/ bmjopen-2020-042384

- Prepublication history and additional material for this paper is available online. To view these files, please visit the journal online (http://dx.doi.org/10. 1136/bmjopen-2020-042384).

Received 04 July 2020 Revised 17 January 2021 Accepted 18 January 2021

Check for updates

(c) Author(s) (or their employer(s)) 2021. Re-use permitted under CC BY-NC. No commercial re-use. See rights and permissions. Published by BMJ.

For numbered affiliations see end of article.

Correspondence to Professor Xue-Song Mi; mxsong@163.com and Professor Wai-kit Ming; wkming@connect.hku.hk

\section{ABSTRACT}

Objective To determine the effects of the intraocular injection of antivascular endothelial growth factor (antiVEGF) drugs on the refractive status of infants with retinopathy of prematurity (ROP).

Design Systematic review and meta-analysis of the refractive status of infants with ROP who receive anti-VEGF drugs.

Data sources The PubMed, Web of Science and Embase databases and the ClinicalTrials.gov website were searched up to June 2020.

Eligibility criteria when selecting studies We included randomised controlled trials (RCTs) and observational studies that compared refractive errors between anti-VEGF drug and laser therapies.

Data extraction and synthesis Data extraction and riskof-bias assessments were conducted by two independent reviewers. We used a random-effect model to pool outcomes. The outcome measures were the spherical equivalents, axial length $(\mathrm{AL})$, anterior chamber depth (ACD) and lens thickness (LT).

Results Thirteen studies involving 1850 eyes were assessed: 914 in the anti-VEGF drug group, and 936 in the control (laser) group. Children who received anti-VEGF drug treatment had less myopia than those who received laser therapy (mean difference $=1.80 \mathrm{D}, 95 \% \mathrm{Cl} 0.97$ to 2.63, $p<0.0001, I^{2}=78 \%$ ). The AL, ACD and LT did not reach statistical significance difference between the two groups. The current evidence indicates that the refractive safety in children with ROP is better for anti-VEGF drug treatment than for laser therapy.

Conclusions This meta-analysis indicates that antiVEGF drug therapy results in less myopia compared with laser therapy. However, there are relatively few published articles on refractive errors in ROP, and so high-quality and powerful RCTs are needed in the future.

PROSPERO registration number CRD42020160673.

\section{INTRODUCTION}

Retinopathy of prematurity (ROP) is a common blinding disease among children in developed countries and is becoming increasingly popular in developing countries. ${ }^{1}$ Characterised by retinal ischaemia, aberrant
Strengths and limitations of this study

- Our meta-analysis adhered to the methodology recommendations of the Cochrane Handbook. We conducted a thorough literature search.

- The article describes a review protocol that is formally registered on PROSPERO, and the study was conducted and reported on using rigorous methods following the Preferred Reporting Items for Systematic Reviews andMeta-Analyses statement.

- We included other parameters that may affect the refractive errors in children with retinopathy of prematurity in our meta-analysis, such as anterior chamber depth, lens thickness and axial length.

- The refractive error measures were from different follow-up time points across studies; this may confound the evaluation of refractive error differences between antivascular endothelial growth factor and laser.

- Most of the included studies had an observational design, with only two randomised controlled trials (RCTs) being included, The inclusion of more RCTs would have allowed more-reliable conclusions to be drawn.

angiogenesis, fibrovascular proliferation and progressive vitreoretinal traction, ROP accounts for $14 \%$ and $20 \%$ of cases of childhood blindness in the USA and developing countries, respectively. ${ }^{2}$

ROP is a unique retinal vascular proliferative disease occurring in premature and low birthweight infants. ${ }^{3}$ Retinal vascularisation normally occurs at around 12 weeks of gestation and is completed by $36-40$ weeks. This prolonged development period means that the retinal system is immature when infants leave the uterus prematurely. The loss of the maternal interaction environment and exposure to high oxygen levels in premature infants can lead to the cessation of retinal vascularisation, damage to the capillary endothelium, hypoxia of the 
retinal blood vessels, and stimulation of fibrovascular tissue proliferation, and might even finally lead to traction retinal detachment. $^{4}$

Laser photocoagulation has previously been the mainstay treatment for ROP. While this intervention is effective and safe, a few defects can remain, such as high myopia, visual field loss and retinal destruction. An intensive study of ROP found that the levels of vascular endothelial growth factor (VEGF) were markedly elevated in the vitreous of eyes at stage- 4 ROP. ${ }^{5}$ In a normally developing retina, VEGF promotes the development of blood vessels from the optic nerve to the periphery, whereas the overexpression of VEGF in preterm infants leads to abnormal vascular proliferation. ${ }^{6}$ This situation has prompted researchers to use anti-VEGF drugs to treat ROP. Many studies have shown that the intravitreal injection of anti-VEGF drugs may be an effective clinical intervention for ROP. ${ }^{7-9}$ However, the effects of this intervention are relatively short term, while its long-term complications remain unclear, such as postoperative refractive errors. Kang et $a l^{10}$ showed that anti-VEGF drugs do not cause refractive errors after ROP treatment, while Kabataş et $a l^{11}$ found that effects of the intravitreal injection of anti-VEGF drugs did not differ significantly from those of laser photocoagulation, with both potentially causing refractive errors.

The increasing clinical application of anti-VEGF drugs makes it important to know whether these drugs can also cause refractive errors in children with ROP. Hence, the purpose of the present meta-analysis was to determine the effects of anti-VEGF drugs on the refractive status of ROP compared with laser treatment, and to verify their clinical safety. The outcome measures considered in this study were the spherical equivalents (SE), axial length (AL), anterior chamber depth (ACD) and lens thickness (LT).

\section{METHODS}

Our study is reported on here in accordance with the PRISMA guidelines for meta-analyses. ${ }^{12}$

\section{Data sources and search strategy}

From their inceptions to January 2020, we searched the PubMed, Web of Science, Embase databases and the ClinicalTrials.gov website using keywords and medical subject headings. Only studies reported on in English were considered for inclusion. We also searched the reference lists of the selected articles to identify any other relevant articles. The search terms included "retinopathy of prematurity," "prematurity retinopathy," "retrolental fibroplasia," "fibroplasia, retrolental," "ROP," "anti-VEGF," "bevacizumab," "Avastin," "Lucentis," "ranibizumab," "aflibercept," "anti-vascular endothelial growth factor," "Mvasi," and "refractive errors," "disorders, refractive," and "ametropias." The search strategy is detailed in the S1 strategy (online supplemental material).

\section{Study selection and eligibility criteria}

Each study was independently screened by two of the authors (QK and X-SM), with discrepancies between them resolved through discussion with the third author (W-kM). The following inclusion criteria were applied : (1) children who had been clearly diagnosed with ROP, (2) subjects in the intervention group had received an intraocular injection of an anti-VEGF drug that can be used in children with ROP, (3) subjects in the control group had received treatment of the eye using a retinal argon or diode laser, (4) the outcome of interest was the refractive status of the treated children with ROP, including SE and ocular biometric structural features such as AL, ACD and LT, and (5) the study design was a randomised controlled trial (RCT) or an observational study. We excluded children with stage-4 ROP, stage-5 ROP or other eye diseases such as congenital cataract or glaucoma prior to treatment.

\section{Data extraction and quality assessment}

The following information was extracted for the included studies: name of the first author, publication year, sample size, number of eyes, gestational age, birth weight, follow-up time, type of anti-VEGF drug, dose of anti-VEGF drug, and results data (SE, AL, ACD and LT). When two anti-VEGF drugs had been applied in a study, we extracted the data separately and compared the data with the control group.

We entered the extracted data into an Excel file. Two of the authors (QK and X-SM) assessed the quality of studies using the Newcastle-Ottawa Scales (NOS) ${ }^{13}$ The NOS consists of four items for subject selection (maximum four points), one item for comparability between groups (maximum two points), and three items for outcome measurement (maximum three points). The maximum score is therefore nine points, with studies considered to be of moderate quality having scores of 4-6, and those of high quality having scores of $7-9 .^{14}$

\section{Statistical analysis}

The weighted mean differences with 95\% CIs were calculated for continuous variables. Heterogeneity between the included studies was assessed using the $\mathrm{I}^{2}$ statistic. $\mathrm{I}^{2}$ values of $25 \%-50 \%, 50 \%-75 \%$ and $75 \%-100 \%$ were considered to indicate low, moderate and high heterogeneity, respectively. ${ }^{15}$ Due to the possibility of heterogeneity being present between studies, we used a more conservative version of the randomeffect model.

A visual funnel plot was used to evaluate publication bias, with an asymmetric plot indicating that publication bias was present. Egger's test was further used to provide accurate assessments of publication bias, with if $\mathrm{p}<0.05$, considered to indicate some degree of publication bias.

All statistical analyses were performed using RevMan software (V.5.3, Nordic Cochrane Centre) and Stata software (V.12.0, Stata Corporation).

\section{RESULTS}

\section{Literature search}

The initial literature search identified 121 records. After screening the titles and abstracts, 31 potentially eligible studies were assessed for inclusion. After reading the full texts, 13 studies were finally included in the present 

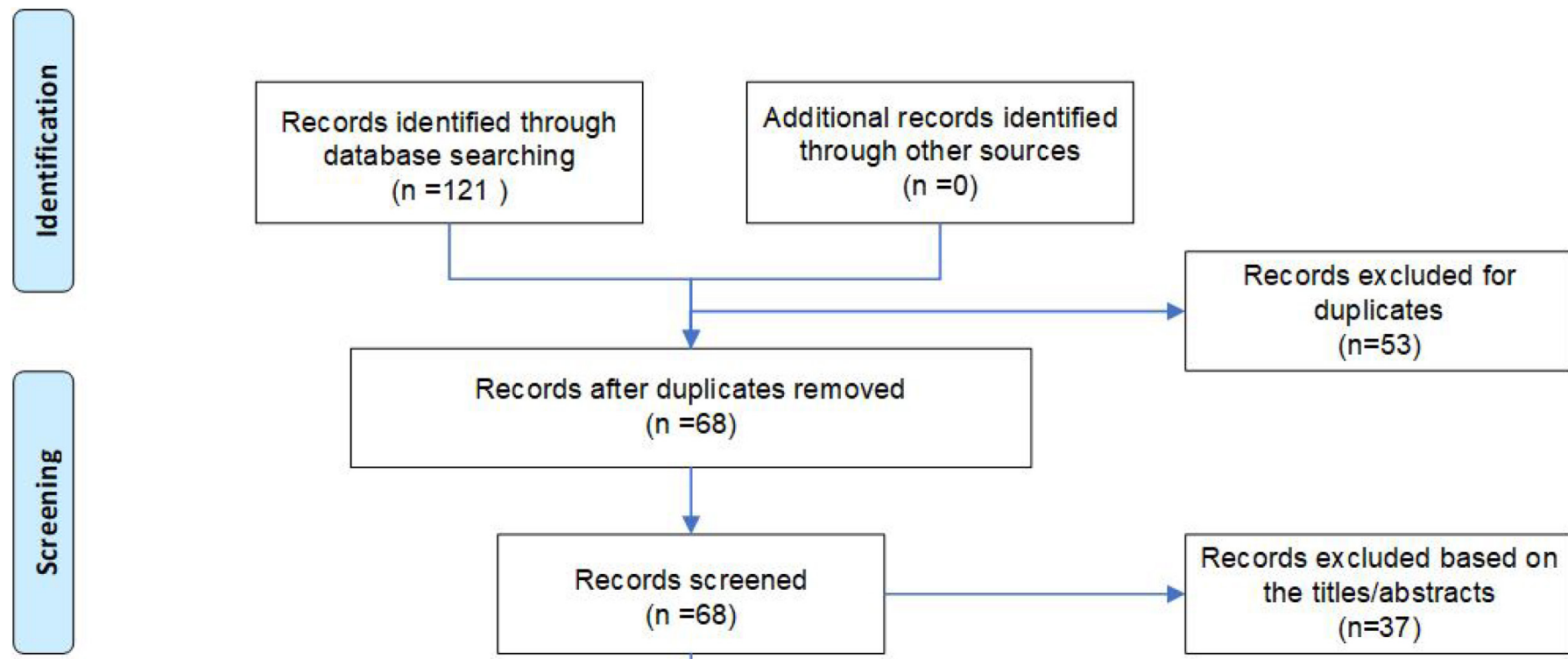

Records after duplicates removed

$(n=68)$
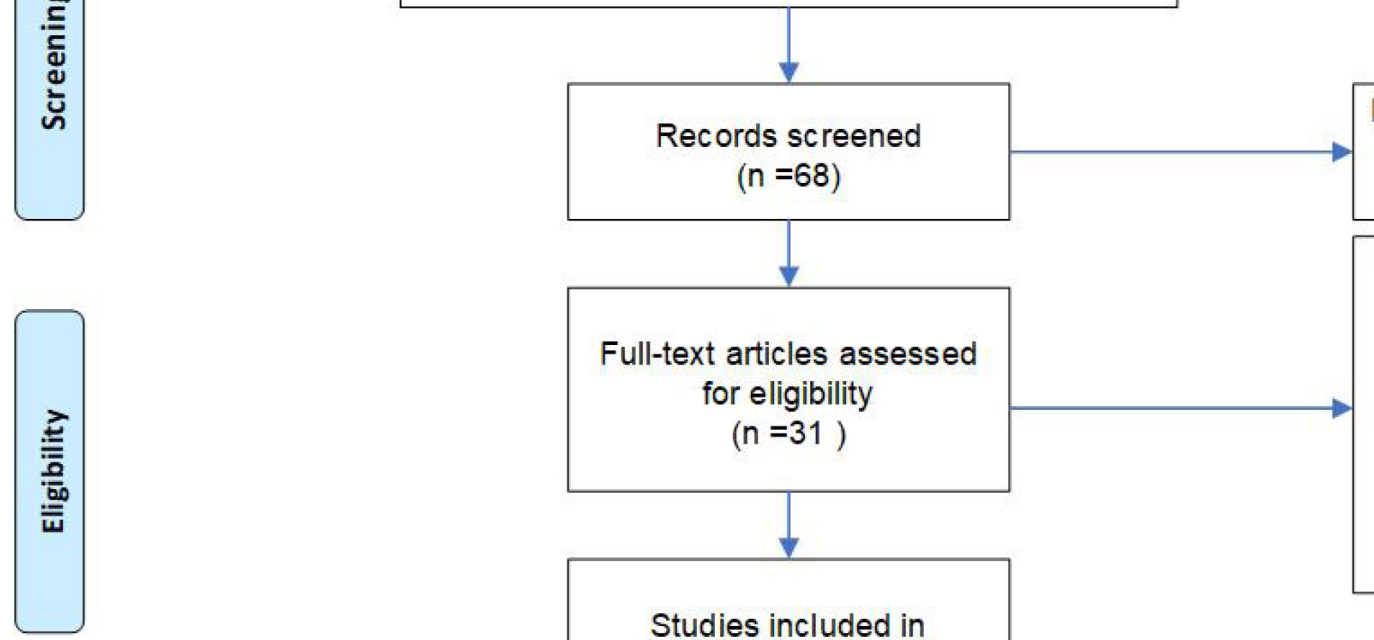

Records excluded based on

the titles/abstracts $(n=37)$
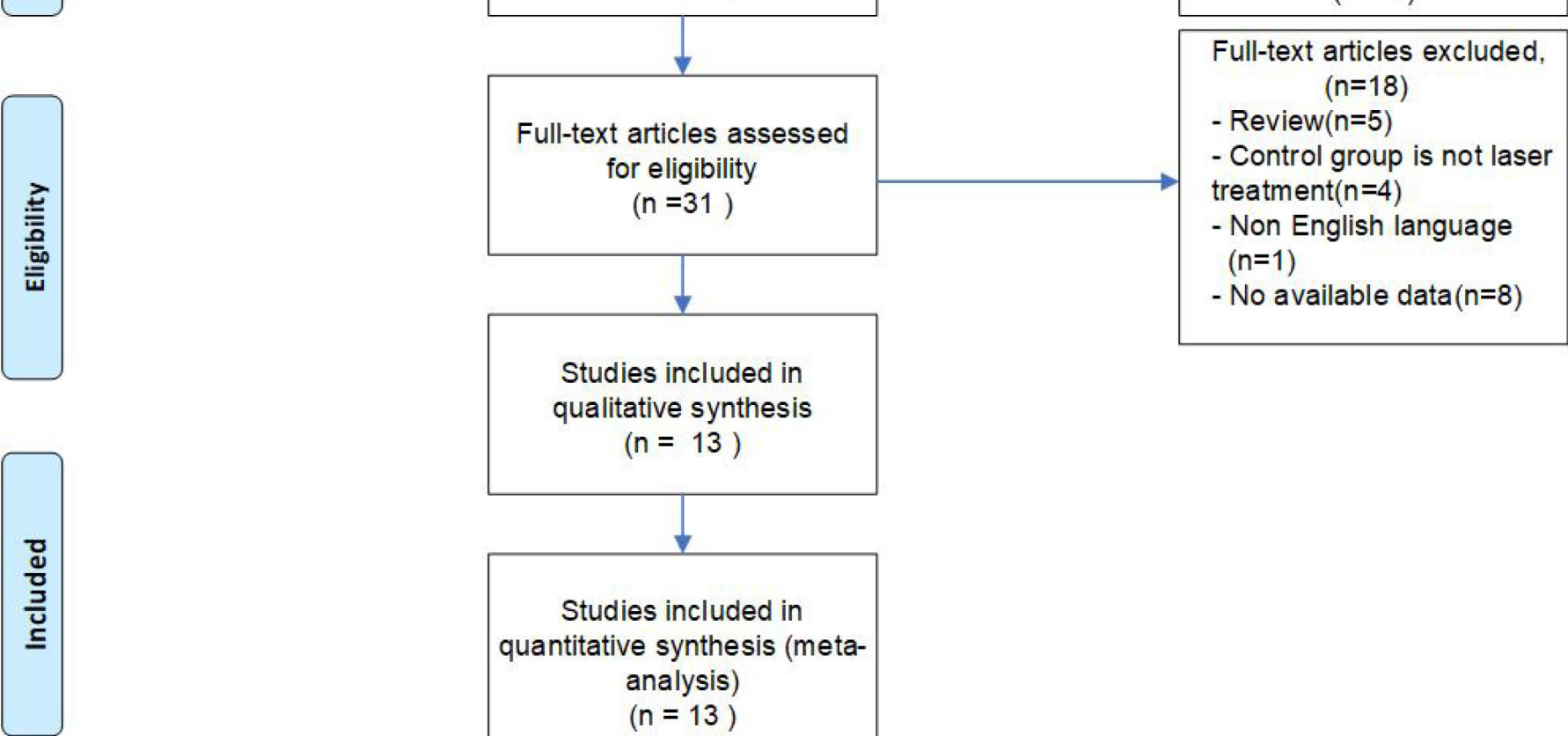

Studies included in

qualitative synthesis

$(n=13)$

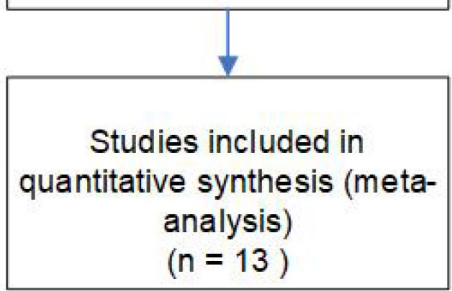

Figure 1 Selection of studies for the meta-analysis.

meta-analysis. The study selection process is illustrated in figure 1 .

The 13 selected studies comprised 2 RCTs, ${ }^{16} 17$ and 11 observational studies. ${ }^{11}{ }^{18-27}$ According to the scoring criteria of the NOS, 11 studies were evaluated as being of high quality, while 2 were evaluated as being of moderate quality. The NOS score of the included studies ranged from 6 to 8 , with a median of 8 . All of the articles had been published between 2013 and 2019. The sample sizes in the studies ranged from 12 to 397 , with a total of 1850 eyes: 914 in the anti-VEGF drug group and 936 in the control group. The included anti-VEGF drugs were bevacizumab and ranibizumab, with one of these drugs administered in 12 studies and 2 drugs administered in 1 study. The dose of anti-VEGF drugs also varied among the included studies, from a minimum of $0.2 \mathrm{mg}$ to a maximum of was $1.25 \mathrm{mg}$, with most of the studies using $0.625 \mathrm{mg}$.

After injecting anti-VEGF drugs, children with ROP were followed regularly for more than 6 months, ranging from 9 months to 5 years. SE values were reported for all of the 13 included studies, while ALs, ACDs and LTs were reported for 4, 3 and 2 studies, respectively. We presented the main information within the included studies in our meta-analysis (table 1).

\section{Main outcomes}

Spherical equivalent

The SE values were reported for 914 eyes in the antiVEGF drug group and 936 eyes in the control group (figure 2). The SE values were higher in the anti-VEGF drug group than in the control group $(\mathrm{MD}=1.80 \mathrm{D}, 95 \%$ CI 0.97 to 2.63$)$, with a high heterogeneity $\left(\mathrm{I}^{2}=78 \%\right)$. The 


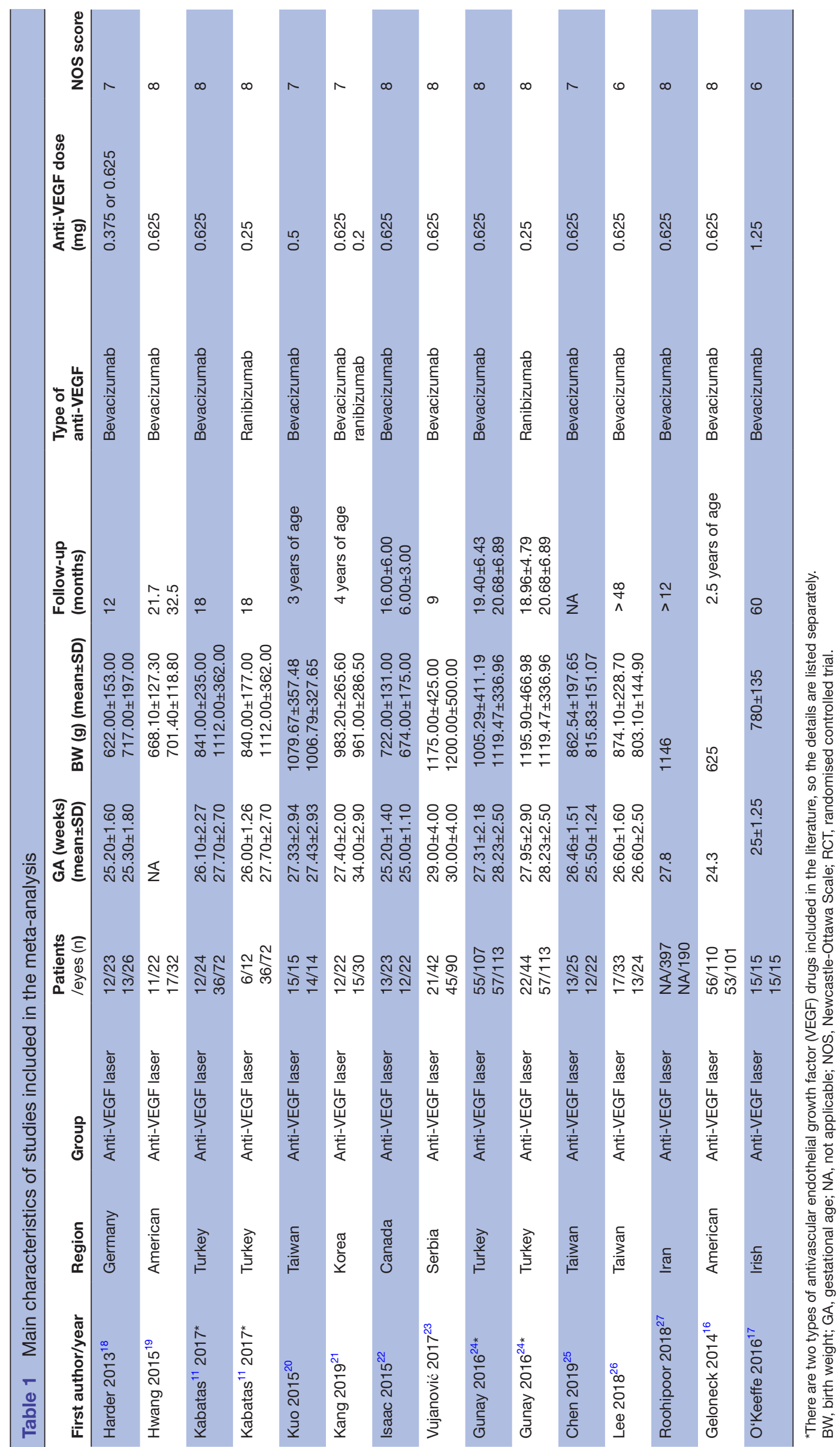




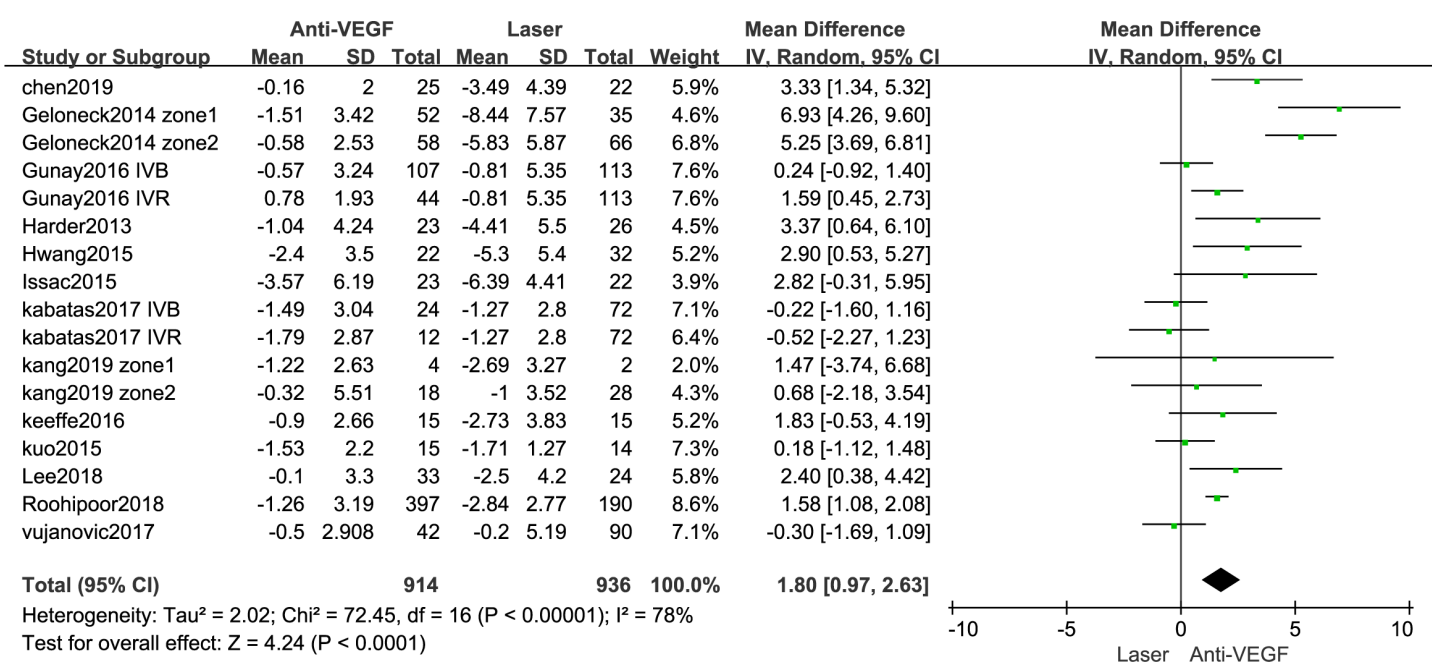

Figure 2 Forest plot of spherical equivalent. VEGF, vascular endothelial growth factor.

findings of the subgroup analysis of the SE according to type of article are summarised in figure 3. At the same time, according to different types of anti-VEGF drugs(online supplemental material S2 forest plot) and different follow-up time (online supplemental material S3 forest plot), we conducted a subgroup analysis.

\section{Axial length}

Three articles ${ }^{23} 2526$ reported the AL, with 251 eyes in the anti-VEGF drug group and 362 eyes in the control group (figure 4). There was no significant difference in the $\mathrm{AL}$ between the groups ( $\mathrm{MD}=-0.04 \mathrm{~mm}, 95 \% \mathrm{CI}-0.30$ to $0.21)$, and the heterogeneity was low $\left(\mathrm{I}^{2}=30 \%\right)$.
Anterior chamber depth

Three articles ${ }^{23} 2526$ reported the ACD in children with ROP who were or were not taking anti-VEGF drugs. We found no difference in the ACD between the anti-VEGF drug and control groups $(\mathrm{MD}=0.19 \mathrm{~mm} ; 95 \% \mathrm{CI}-0.14$ to $0.52, \mathrm{I}^{2}=85 \%$; figure 5 ). There was high heterogeneity $\left(\mathrm{I}^{2}=85 \%\right)$, but excluding Vujanović's study in the sensitivity analysis resulted in moderate heterogeneity ( $\mathrm{MD}=0.39 \mathrm{~mm} ; 95 \% \mathrm{CI}-0.06$ to $0.84, \mathrm{I}^{2}=64 \%$ ).

\section{Lens thickness}

Two articles ${ }^{23} 25$ reported the LT, which did not differ significantly between the anti-VEGF drug and laser groups

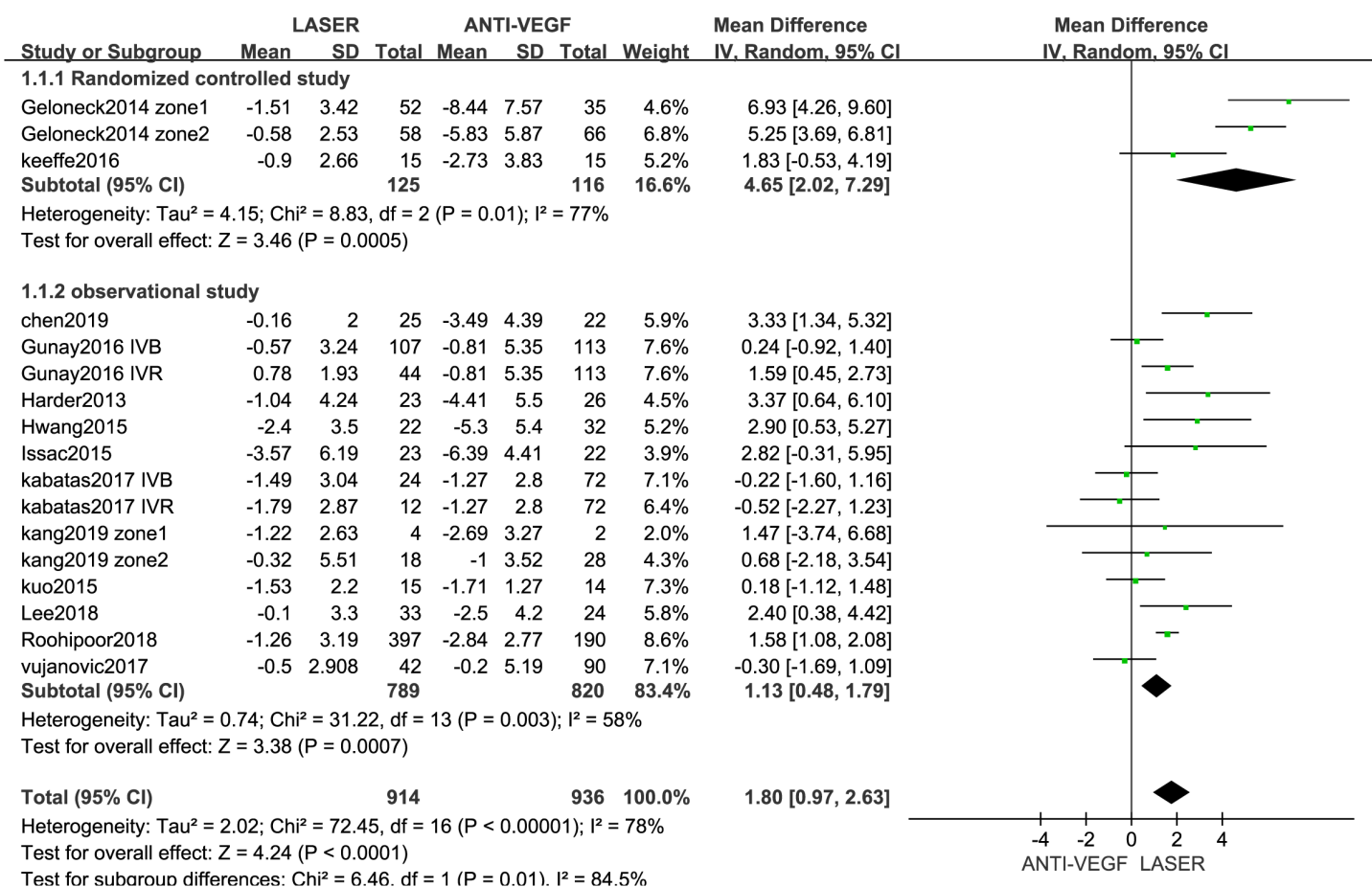

Figure 3 Forest plot of the effect antivascular endothelial growth factor (VEGF) therapy on spherical equivalent, according to the types of article included. 


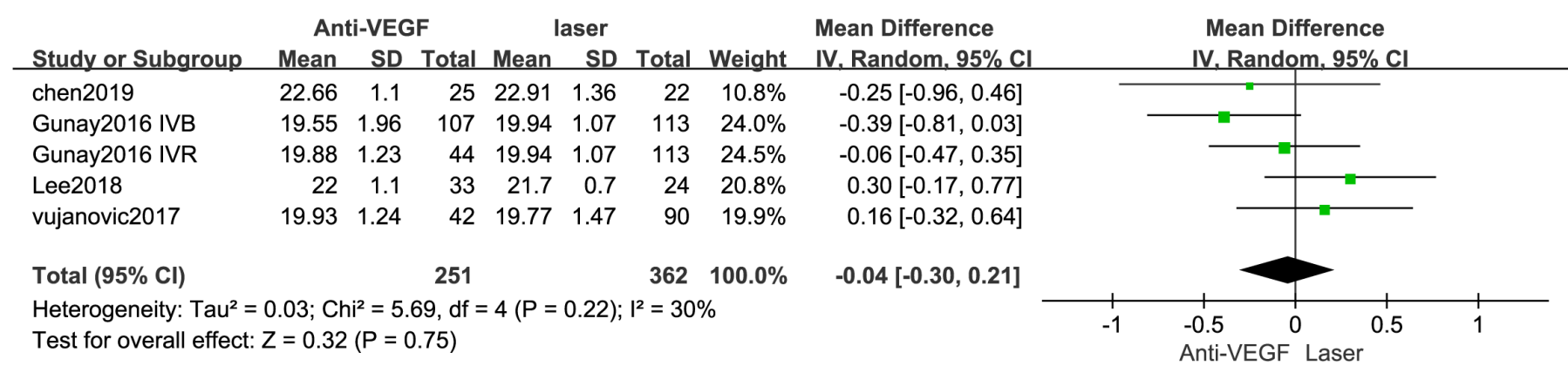

Figure 4 Forest plot of axial length (AL). VEGF, vascular endothelial growth factor.

$(\mathrm{MD}=0.06 \mathrm{~mm} ; 95 \% \mathrm{CI}-0.56$ to $0.67, \mathrm{p}=0.85)$, and $\mathrm{I}^{2}$ was 97\% (online supplemental material: S4 forest plot).

\section{Publication bias}

Visual examinations of funnel plots constructed using Stata software and also statistical calculations using in Egger's test did not reveal any publication bias $(\mathrm{p}=0.401)$. (online supplemental material S5 funnel plot)

\section{DISCUSSION}

The present meta-analysis identified that 13 previous studies have investigated the association between treatments and refractive errors among children with ROP, and analysed SE, AL, ACD and LT. A significant difference in SE was found between the two study groups. This means that anti-VEGF drug treatment reduces the degree of myopia in children with ROP compared with laser treatment, as consistently found in both the comprehensive and subgroup analyses. However, no significant differences were found in the other variables analysed in this study.

Meta-analyses of similar subjects have also been reported. ${ }^{28}$ The meta-analysis of Tan et al included 7 articles covering 519 eyes, but this was limited to the antiVEGF drug bevacizumab. Although the main finding of our meta-analysis was consistent with previous metaanalyses, there are some differences between them. First, our study analysed the largest amount of data (13 articles covering 1850 eyes) and included some recently published literature, which increased the statistical power of the analyses. Second, in addition to the antiVEGF drug bevacizumab, another anti-VEGF drug that is commonly used in clinical practice was also included (ranibizumab), which makes the present conclusions closer to clinical reality. Third, we added other ocular parameters to investigate how anti-VEGF drug and laser therapies affect refractive errors: ACD, LT and AL. A previous study showed that these ocular refractive parameters may be related to myopic adults with $\mathrm{ROP},{ }^{29}$ but no evidence was provided for laser treatment and anti-VEGF drug treatment exerting different effects on the refractive status in children with ROP. We, therefore, analysed these ocular parameters in laser and anti-VEGF drug groups, with the obtained results providing further evidence that anti-VEGF drug treatment is safe for children with ROP.

This meta-analysis of 13 articles synthesised the literature to evaluate the refractive safety of anti-VEGF drugs for children with ROP and has shown that anti-VEGF drug treatment provides better refractive results than does laser treatment. As seen in previous studies, the degree of myopia was reduced more by anti-VEGF drug therapy than by laser treatment in the current study. Most previous studies have quantified refractive errors using SE values, since this parameter is considered the primary measure of such errors, and so we also used this parameter to explore group differences .

Kuo $e t a l^{20}$ and Issec $e t a l^{22}$ reported that refractive errors did not differ significantly between anti-VEGF drug and laser groups. However, our meta-analysis found that anti-VEGF drug therapy reduces refractive errors more than does laser treatment. Two factors may explain this difference: (1) both of the previous studies included small samples, and (2) a higher proportion of the children included in the present study had severe ROP. The present findings indicate that anti-VEGF drug therapy may be an alternative to laser therapy for reducing refractive errors in children with ROP. Our subgroup analysis found that anti-VEGF drug therapy exerted better effects on refractive errors than did laser therapy, based on findings in both RCTs and observational studies.

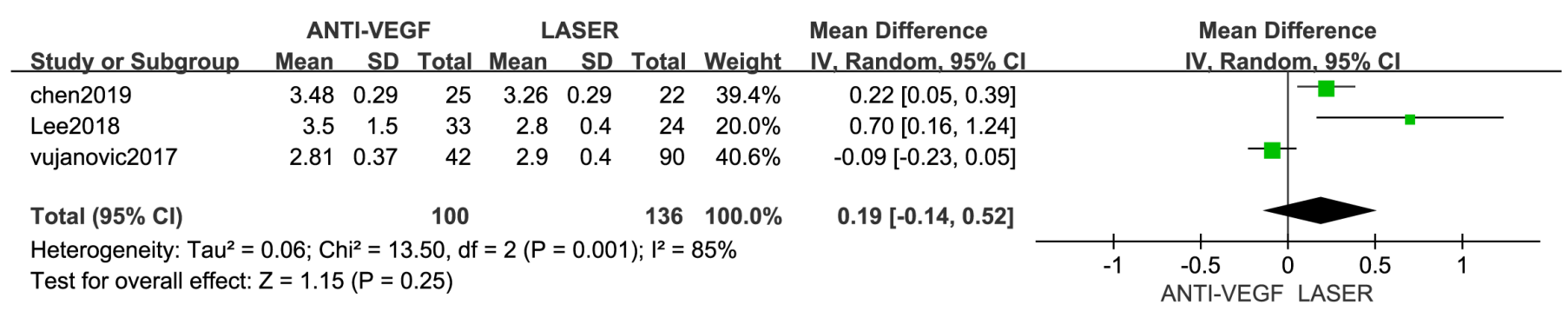

Figure 5 Forest plot of anterior chamber depth (ACD). VEGF, vascular endothelial growth factor. 
Laser therapy has been considered the first choice of treatment for ROP and has well-established safety and efficacy. However, this approach results in the retina being permanently cauterised, leading to inadequate vascularisation and the risk of visual field loss, high myopia and cataracts. Regarding refraction, it has also been reported that laser treatment may be a risk factor for refractive errors in children with ROP. ${ }^{30}$ Therefore, the impact of future damage to the refractive status that may result from laser treatment needs to be considered when treating children with ROP, indicating the need to find a treatment method with increased effectiveness and safety. A previous in-depth study of the ROP mechanism found that the intraocular injection of an anti-VEGF drug may be a good alternative to laser treatment. ${ }^{22}$ The intraocular injection of anti-VEGF drugs has the advantages of less trauma and pain and involving an easy procedure, which has resulted in it being increasingly used by a large number of clinicians. This situation makes it essential to clarify the safety regarding refractive errors as soon as possible, and the present meta-analysis has provided good evidence for the refractive safety of anti-VEGF drugs.

Changes in the biometric structure of the eye-such as in ACD, LT, AL, corneal curvature and corneal diameter-may be related to increased refractive errors. ${ }^{31-33}$ We, therefore, regard it as essential to consider the above indicators of ocular biometric structure when verifying the refractive status of children with ROP. Although there have been previous meta-analyses of refractive outcomes after treatment of ROP with anti-VEGF drug therapy, none of these meta-analyses explored the relationship between refractive outcomes and the biometric structure of the eye when comparing anti-VEGF drug and laser therapies. Our study found no significant intergroup differences in these parameters. There is also considerable debate about whether anti-VEGF drug treatment of ROP will induce changes in ocular parameters. Lee et al found that AL did not differ among different treatment groups. Gunay et $a l^{34}$ reported that in children who receive anti-VEGF drug therapy, the AL might be related to the development of myopia and is not related to the ACD or LT. The Bevacizumab Eliminates the Angiogenic Threat for ROP (BEAT-ROP) believes that anti-VEGF drug treatment may facilitate the continuation of the local growth factor expression and signalling pathways, allowing the anterior segment to develop normally. ${ }^{16}$ The small number of articles that have reported on the ocular biometric structure makes it difficult to draw definitive conclusions, and so more high-quality RCTs are needed to verify the impact of anti-VEGF drugs on the ocular biometric structure.

We consider that future research should focus on two main aspects. First, there is no unified standard for the optimal dose of anti-VEGF drugs to use in the treatment of ROP. Most clinical applications used doses are half of the adults, but if other doses of anti-VEGF treatment of ROP affect the result, we do not know, so in the future, a clear plan for the dose of anti-VEGF needs to be proposed. Second, there is no clear standard for the follow-up time of children with ROP. The conclusions that may be drawn lack credibility if the follow-up time is too short, and so the most-appropriate follow-up time of children after treatment with anti-VEGF drugs also needs to be determined.

The first strength of our meta-analysis is that it adhered to the methodology recommendations of the Cochrane Handbook, and included conducting a thorough literature search. Second, this meta-analysis has a formally registered review protocol on PROSPERO, and our investigations were conducted and reported with rigorous methods following the PRISMA statement. Third, our meta-analysis included other parameters that may affect the refractive errors in children with ROP, such as ACD, LT and AL. The results further strengthen the evidence for the safety of anti-VEGF drugs in children with ROP. However, our study also had certain limitations. First, the refractive error measures were from different follow-up time points across studies; this may confound the evaluation of refractive error differences between anti-VEGF and laser. Second, most of the included studies had an observational design, with only two RCTs being included, The inclusion of more RCTs would have allowed more reliable conclusions to be drawn.

\section{CONCLUSIONS}

In conclusion, the present meta-analysis has shown that anti-VEGF drug therapy reduces the degree of myopia more effectively than does laser treatment. The current evidence indicates that anti-VEGF drug treatment has better refractive safety than laser therapy for children with ROP. Since intraocular injections of angiogenesis factor inhibitors are increasingly being applied, more high-quality RCTs are required.

\section{Author affiliations}

${ }^{1}$ Department of Ophthalmology, the First Affiliated Hospital of Jinan University, Guangzhou, China

${ }^{2}$ Department of Public Health and Preventive Medicine, School of Medicine, Jinan University, Guangzhou, China

${ }^{3}$ Li Ka Shing Faculty of Medicine, University of Hong Kong, Hong Kong, China ${ }^{4}$ Changsha Academician Expert Workstation, Aier Eye Hospital Group, Changsha, China

Contributors QK and X-SM conceived the idea of the article. QK and X-SM did the literature search. All authors undertook data acquisition and analysis. QK carried out the manuscript preparation. X-SM and W-kM were responsible for the revision of the manuscript. All authors have read and approved the final manuscript.

Funding The study is supported by the National NSFC (82074169), Hygiene \& Health Appropriated Technology and Promoting Project of Guangdong Province (202006130025341204, 201905270933056876) and Project of Administration of Traditional Chinese Medicine of Guangdong Province of China (20202045).

Competing interests None declared.

Patient consent for publication Not required.

Provenance and peer review Not commissioned; externally peer reviewed.

Data availability statement All data relevant to the study are included in the article or uploaded as supplementary information. 
Supplemental material This content has been supplied by the author(s). It has not been vetted by BMJ Publishing Group Limited (BMJ) and may not have been peer-reviewed. Any opinions or recommendations discussed are solely those of the author(s) and are not endorsed by BMJ. BMJ disclaims all liability and responsibility arising from any reliance placed on the content. Where the content includes any translated material, BMJ does not warrant the accuracy and reliability of the translations (including but not limited to local regulations, clinical guidelines, terminology, drug names and drug dosages), and is not responsible for any error and/or omissions arising from translation and adaptation or otherwise.

Open access This is an open access article distributed in accordance with the Creative Commons Attribution Non Commercial (CC BY-NC 4.0) license, which permits others to distribute, remix, adapt, build upon this work non-commercially, and license their derivative works on different terms, provided the original work is properly cited, appropriate credit is given, any changes made indicated, and the use is non-commercial. See: http://creativecommons.org/licenses/by-nc/4.0/.

\section{ORCID iD}

Xue-Song Mi http://orcid.org/0000-0001-9146-1715

\section{REFERENCES}

1 Chen J, Smith LEH. Retinopathy of prematurity. Angiogenesis 2007;10:133-40.

2 Tran KD, Cernichiaro-Espinosa LA, Berrocal AM. Management of Retinopathy of Prematurity--Use of Anti-VEGF Therapy. Asia Pac J Ophthalmol 2018;7:56-62.

3 Bashinsky AL. Retinopathy of prematurity. N C Med J 2017;78:124-8.

4 Hellström A, Smith LEH, Dammann O. Retinopathy of prematurity. Lancet 2013;382:1445-57.

5 Lashkari K, Hirose T, Yazdany J, et al. Vascular endothelial growth factor and hepatocyte growth factor levels are differentially elevated in patients with advanced retinopathy of prematurity. Am J Pathol 2000;156:1337-44.

6 Sankar MJ, Sankar J, Chandra P. Anti-Vascular endothelial growth factor (VEGF) drugs for treatment of retinopathy of prematurity. Cochrane Database Syst Rev 2018;1:Cd009734.

7 Walz JM, Bemme S, Pielen A, et al. The German ROP registry: data from 90 infants treated for retinopathy of prematurity. Acta Ophthalmol 2016;94:e744-52.

8 Tawse KL, Jeng-Miller KW, Baumal CR. Current practice patterns for treatment of retinopathy of prematurity. Ophthalmic Surg Lasers Imaging Retina 2016;47:491-5.

9 VanderVeen DK, Melia M, Yang MB, et al. Anti-Vascular endothelial growth factor therapy for primary treatment of type 1 retinopathy of prematurity: a report by the American Academy of ophthalmology. Ophthalmology 2017;124:619-33.

10 Kang HG, Choi EY, Byeon SH, et al. Anti-Vascular endothelial growth factor treatment of retinopathy of prematurity: efficacy, safety, and anatomical outcomes. Korean J Ophthalmol 2018;32:451-8.

11 Kabataș EU, Kurtul BE, Altıaylık Özer P, et al. Comparison of intravitreal bevacizumab, intravitreal ranibizumab and laser photocoagulation for treatment of type 1 retinopathy of prematurity in Turkish preterm children. Curr Eye Res 2017;42:1054-8.

12 Moher D, Liberati A, Tetzlaff J, et al. Preferred reporting items for systematic reviews and meta-analyses: the PRISMA statement. BMJ 2009;339:b2535.

13 Wells GA SD, O'Connell D, Peterson J. The Newcastle-Ottawa scale (NOS) for assessing the quality of nonrandomised studies in meta-analyses. ohrica, 2000. Available: http://wwwohrica/programs/ clinical_epidemiology/oxfordasp [Accessed 25 Jul 2020].

14 Gu W-J, Hou B-L, Kwong JSW, et al. Association between intraoperative hypotension and 30-day mortality, major adverse cardiac events, and acute kidney injury after non-cardiac surgery: a meta-analysis of cohort studies. Int J Cardiol 2018;258:68-73.

15 Higgins JPT, Thompson SG, Deeks JJ, et al. Measuring inconsistency in meta-analyses. BMJ 2003:327:557-60.

16 Geloneck MM, Chuang AZ, Clark WL, et al. Refractive outcomes following bevacizumab monotherapy compared with conventional laser treatment: a randomized clinical trial. JAMA Ophthalmol 2014;132:1327-33.

17 O'Keeffe N, Murphy J, O'Keefe M, et al. Bevacizumab compared with diode laser in stage 3 posterior retinopathy of prematurity: a 5 year follow up. Ir Med J 2016;109:355.

18 Harder BC, Schlichtenbrede FC, von Baltz S, et al. Intravitreal bevacizumab for retinopathy of prematurity: refractive error results. Am J Ophthalmol 2013;155:1119-24.

19 Hwang CK, Hubbard GB, Hutchinson AK, et al. Outcomes after intravitreal bevacizumab versus laser photocoagulation for retinopathy of prematurity: a 5-year retrospective analysis. Ophthalmology 2015;122:1008-15

20 Kuo H-K, Sun I-T, Chung M-Y, et al. Refractive error in patients with retinopathy of prematurity after laser photocoagulation or bevacizumab monotherapy. Ophthalmologica 2015;234:211-7.

21 Kang HG, Kim TY, Han J, et al. Refractive outcomes of 4-year-old children after intravitreal anti-vascular endothelial growth factor versus laser photocoagulation for retinopathy of prematurity. Korean J Ophthalmol 2019;33:272-8.

22 Isaac M, Mireskandari K, Tehrani N. Treatment of type 1 retinopathy of prematurity with bevacizumab versus laser. J Aapos 2015;19:140-4.

23 Vujanovic M, Stankovic-Babic G, Oros A, et al. Refractive errors in premature infants with retinopathy of prematurity after anti-vascular endothelial growth factor (anti-VEGF) therapy. Vojnosanit Pregl 2017;74:323-8.

24 Gunay M, Sukgen EA, Celik G, et al. Comparison of bevacizumab, ranibizumab, and laser photocoagulation in the treatment of retinopathy of prematurity in turkey. Curr Eye Res 2017;42:462-9.

25 Chen Y-C, Chen S-N. Foveal microvasculature, refractive errors, optical biometry and their correlations in school-aged children with retinopathy of prematurity after intravitreal antivascular endothelial growth factors or laser photocoagulation. Br J Ophthalmol 2020;104:691-6.

26 Lee Y-S, See L-C, Chang S-H, et al. Macular structures, optical components, and visual acuity in preschool children after intravitreal bevacizumab or laser treatment. Am J Ophthalmol 2018;192:20-30.

27 Roohipoor R, Karkhaneh R, Riazi-Esfahani M, et al. Comparison of intravitreal bevacizumab and laser photocoagulation in the treatment of retinopathy of prematurity. Ophthalmol Retina 2018;2:942-8.

28 Tan Q-Q, Christiansen SP, Wang J. Development of refractive error in children treated for retinopathy of prematurity with anti-vascular endothelial growth factor (anti-VEGF) agents: a meta-analysis and systematic review. PLoS One 2019;14:e0225643.

29 Baker PS, Tasman W. Myopia in adults with retinopathy of prematurity. Am J Ophthalmol 2008;145:1090-4.

30 Al-Otaibi AG, Aldrees SS, Mousa AA. Long term visual outcomes in laser treated threshold retinopathy of prematurity in central Saudi Arabia. Saudi J Ophthalmol 2012;26:299-303.

31 Cook $\mathrm{A}$, White $\mathrm{S}$, Batterbury $\mathrm{M}$, et al. Ocular growth and refractive error development in premature infants with or without retinopathy of prematurity. Invest Ophthalmol Vis Sci 2008;49:5199-207.

32 Chen T-C, Tsai T-H, Shih Y-F, et al. Long-Term evaluation of refractive status and optical components in eyes of children born prematurely. Invest Ophthalmol Vis Sci 2010;51:6140-8.

33 Wu W-C, Lin R-I, Shih C-P, et al. Visual acuity, optical components, and macular abnormalities in patients with a history of retinopathy of prematurity. Ophthalmology 2012;119:1907-16.

34 Gunay M, Sekeroglu MA, Bardak H, et al. Evaluation of refractive errors and ocular biometric outcomes after intravitreal bevacizumab for retinopathy of prematurity. Strabismus 2016;24:84-8. 